\title{
Multiple Injektionen oder Pumpentherapie bei Typ-1-Diabetes?
}

Fragestellung: Schützt die Insulinpumpentherapie Typ-1-Diabetiker langfristig besser vor kardiovaskulären Ereignissen als die intensivierte konventionelle Insulintherapie?

Hintergrund: Bei schlechter Stoffwechseleinstellung und hohem $\mathrm{HbA}_{1 \mathrm{c}}$ drohen Typ-1-Diabetikern kardiovaskuläre Komplikationen. Strenge Blutzuckereinstellung senkt das Risiko, verzögert den Krankheitsbeginn und verringert die Progression. Trotzdem ist einer schwedischen Studie aus dem Jahr 2014 zufolge das Sterberisiko und die kardiovaskuläre Mortalität bei diesen Patienten selbst bei einem $\mathrm{HbA}_{1 \mathrm{c}}$ unter 6,9\% immer noch doppelt so hoch wie in der Allgemeinbevölkerung. Bisher ist unzureichend untersucht, ob die intensivierte konventionelle Insulintherapie und die Insulinpumpentherapie die kardiovaskulären Komplikationen unterschiedlich beeinflussen.

Patienten und Methoden: Im schwedischen Nationalregister sind zwischen 2005 und 2012 insgesamt 18168 Typ-1-Diabetiker erfasst worden. Davon wurden während der vollen Studiendauer 15727 mit multiplen Insulininjektionen und 2441 mit der Insulinpumpe behandelt. Bei der Auswertung wurden 36 Einflussfaktoren berücksichtigt, u.a. me-

\section{Originalie}

Steineck I, Cederholm J, Eliasson B et al. Insulin pump therapy, multiple daily injections, and cardiovascular mortality in 18 168 people with type 1 diabetes: observational study. Brit Med J 2015;350:h3234 dizinische Ausgangsbefunde, medikamentöse Therapien und Begleiterkrankungen, aber auch demografische und soziale Kriterien.

Ergebnisse: Während der Beobachtungsdauer von 6,8 Jahren ereigneten sich 1423 tödliche und nicht tödliche
T1 Kardiovaskuläre Komplikationen bei Spritzen und Pumpe

\begin{tabular}{|c|c|c|c|c|}
\hline & $\begin{array}{l}\text { Insulin- } \\
\text { Injektionen }\end{array}$ & $\begin{array}{l}\text { Insulin- } \\
\text { pumpe }\end{array}$ & $\mathrm{RR}$ & p-Wert \\
\hline \multicolumn{5}{|l|}{ Schwere Endpunkte } \\
\hline Alle koronaren Ereignisse & 10,7 & 6,2 & $-19 \%$ & 0,05 \\
\hline Alle CV Ereignisse & 13,1 & 8,3 & $-12 \%$ & ns \\
\hline CVTodesfälle & 5,1 & 1,8 & $-42 \%$ & 0,005 \\
\hline Gesamtmortalität & 11,0 & 5,3 & $-27 \%$ & 0,007 \\
\hline \multicolumn{5}{|l|}{ Sekundäre Endpunkte } \\
\hline Tödliche koronare Ereignisse & 4,5 & 1,5 & $-45 \%$ & 0,004 \\
\hline Tödliche Schlaganfälle & 0,8 & 0,3 & $-33 \%$ & ns \\
\hline Nicht-CV Mortalität & 5,9 & 3,4 & $-14 \%$ & ns \\
\hline
\end{tabular}

kardiovaskuläre Komplikationen ( Tab. 1). Die Therapie mit der Pumpe führte bei allen untersuchten Endpunkten zu einer stärkeren Reduktion um 12 bis 45\% (meist signifikant) als die Behandlung mit multiplen Injektionen. Die Ergebnisse änderten sich nach Ausschluss von Patienten mit niedrigem BMI und Herzerkrankungen in der Vorgeschichte nicht. Mit der Pumpentherapie wurden 206 Hypoglykämien (148 mit Klinikeinweisung), mit den Injektionen 967 (1366) dokumentiert. Das $\mathrm{HbA}_{\mathrm{lc}}$ lag in beiden Gruppen zu Beginn bei $64 \mathrm{mmol} / \mathrm{mol}(8 \%)$ und fiel während der Studie um 2,1 mmol/mol.

Schlussfolgerung: Bei Typ-1-Diabetikern führt die mehrjährige Insulinpumpentherapie zu einer deutlicheren Abnahme der kardiovaskulären Komplikationen als die intensivierte konventionelle Insulintherapie.

\section{- Kommentar von Prof. Dr. med. Heinrich Holzgreve}

\section{Insulinpumpe sticht multiple Injektionen}

Es handelt sich um eine Beobachtungsstudie auf Bevölkerungsebene. Der Makel dieses Studiendesigns wird durch Berücksichtigung zahlreicher Einflussfaktoren und die hohen Probandenzahlen gemildert, aber nicht beseitigt. Trotzdem: Es handelt sich um die besten bisher verfügbaren Daten und die Autoren haben berechnet, dass nur eine stark wirksame, nicht berücksichtigte Komponente, die bei mindestens $80 \%$ der Probanden vorhanden sein muss, die Ergebnisse neutralisieren könnte.

Da das Ausgangs- $\mathrm{HbA}_{1 c}$ und seine Änderungen im Verlauf in beiden Gruppen identisch waren, kann der Rückgang der kardiovaskulären Komplikationen nicht durch Unterschiede in der langfristigen Stoffwechselkontrolle erklärt werden. Möglicherweise hat die deutlich geringere Zahl von Hypoglykämien un- ter Pumpentherapie viele kardiovaskuläre Komplikationen verhindert.

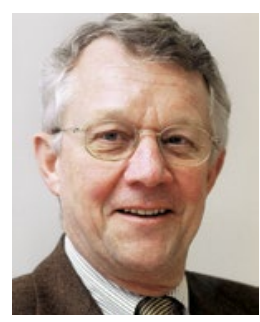

Prof. Dr. med. Heinrich Holzgreve

Internist, Kardiologische Praxis

Burgstr. 7, 80331 München

h.holzgreve@t-online.de 